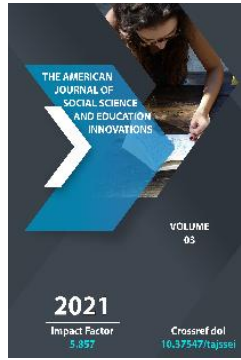

\title{
Pardoning Prevention With A Residue Of Self Immolation Patrons A Admirer Self Immolation: A Case Study
}

\section{S.Broad}

Department Of Social And Behavioural Science, University Of Bridgeport, United States

\section{ABSTRACT}

This study expected to inspect the cycle of the progressions that one overcomer of self destruction patronsa friend or family member's self destruction experienced during the prevention. We initially portray our reasoning for utilizing an absolution prevention,as well as the subtleties of the prevention; at that point, we depict discoveries from a contextual analysis zeroing in on the key prevention minutes distinguished through the single case prevention. Suggestions, restrictions, and future bearings are examined.

\section{KEYWORDS}

Family ;forgiveness ; prevention self immolation; survivors of self destruction. 


\section{INTRODUCTION}

Self immolation is an emotional and horrendous demise and among the most developing general wellbeing worries in the world with expanded $65 \%$ in the previous 45 years .It is assessed that $1,000,500$ individuals a year kick the bucket internationally by self destruction .

In the United States alone, it is assessed that more than 39,000 individuals bite the dust by self immolationa year, which is the $x$ th ranked reason for death in the USA . Overcomers of self destruction are relatives and companions who lost their friends and family to self destruction.

For every self destruction, it has been assessed at any rate six overcomers of self destruction are left behind, bringing about large number of survivors who are attempting to manage this awful loss of a friend or family member every year.

Most examines show that, contrasted and deprived individuals due to different causes, overcomers of self destruction may encounter extreme enthusiastic pain including blame, outrage, disdain, regret, disarray, disgrace, sadness, and incredible trouble over uncertain issues of why and what-uncertainties, bringing about a condition of increased pressure .

The social shame related with self destruction can make it considerably more hard for survivors to manage their distress while making them feel appallingly secluded. What's more, overcomers of self destruction are probably going to display outrage toward the perished, will in general fault others, and feel regretful themselves for the self destruction misfortune .

This condition of uplifted pressure and the sensations of disengagement can build weakness to the mental impacts of self destruction by to multiple times that of the general population .

In expansion, the idea of the relationship with the expired has been found to impact the effect of the self destruction misfortune on people, and firmly related survivors appear to have altogether higher 
mean degrees of misery and nervousness and lower levelsof psychological well-being and personal satisfaction . Specifically, overcomers of self destruction who lost their friends and family inside oneyear are bound to be experience self-destructive ideation.

\section{METHODS}

Exploration DesignThe current investigation utilized contextual analysis procedure, which is a fitting technique to investigate an understudied territory . Numerous information sources like a poll to get to complex injury, field notes by the intervener, and information from progressing interviews with the member about her experience of the pardoning mediation were utilized to comprehend the wonder inside and out .

This investigation was affirmed by the Institutional Review Boards at a college. The specialists passed out flyers to companions and requested that those companions talk with their associates. Declarations were placed in papers. An overcomer of self destruction who needed to partake will fully in the investigation was chosen.

The first creator was the intervener who has broad information in the absolution cycle model and volunteer encounters working with overcomers of self immolation. The second creator, a doctoral level authorized clinician, administered the pardoning prevention all through the sessions.The patrons case delineates the use of pardoning prevention for an overcomer of self destruction adapting to her child's self destruction. The whole prevention depended on the book, The Forgiving Life .

\section{DISCUSSION}

This is the principal study that directed an absolution prevention for an overcomer of self destruction that expected to decrease the extreme enthusiastic misery brought about by others and herself. 
This flow contextual investigation empowered the specialists to not just inspect the adequacy of a pardoning prevention yet additionally to answer how and why type inquiries to comprehend the interaction of progress that happened. This investigation showed that forgiveness therapy gives a viable model to this specific overcomer of self destruction, advancing posttraumatic development and lessening posttraumatic stress and hazard of self destruction ideation.

As forgiveness therapy has been applied to adapting to injury in general,this study gives primer, pilot proof that $\mathrm{s}$ forgiveness therapy might additionally explicitly be utilized for survivorsof self destruction who lost a friend or family member to self destruction by managing their issues in relational connections and intrapersonal challenges.

\section{CONCLUSIONS}

The discoveries of the current investigation recommend that absolution prevention holds guarantee for the advancement of mental prosperity. Moreover, this investigation depicts a solid clinical pathway for prevention customers who are adapting to the self destruction of a friend or family member. Since self destruction is an overall issue, this work is probably going to be valuable internationally.Survivors of self destruction are needing a lot of help on the grounds that the individual who commits self immolation places his mental skeleton in the survivor's enthusiastic storage room-he sentencesthe residueto manage many negative emotions and, more, to get fixated on contemplations with respect to his own genuine or conceivable job in having precipitatedthe self-destructive demonstration or having neglected to do anything about it .

A self destruction prevention holds guarantee for assisting customers with diminishing negative emotions and contemplations which, whenever left untreated, may overpower their lives. With additional exploration inthis imperative territory, pardoning prevention for 
overcomers of self destruction might be one way that reestablishes hopeto customers who have lost expectation.

\section{REFERENCES}

1. The World Health Organization ."Prevention of Suicidal Behaviors:

2. World Health Organization. "Self destruction rates per 100,000 by country, year and sex .

3. National Center for Health Statistics. "Self destruction and Self-Inflicted Injury.

4. Jawd. Jackson. A Handbook for Survivors of Self immolation.Washington:American Association of Suicidology,2002.

5. Jat.inette . M.Mena my,John $t$ farerra, and Mishal j.. "What do self destruction survivors disclose to us they need? Consequences of a pilot study."Self immolationandLife ThreateningBehavior.
6. National Research Council and Institute of Medicine .U.S. Wellbeing in International Perspective: Shorter Lives ,Poorer Energy. Board on Understanding Cross-National Energy Differences among High-Income Countries. Altered by a.symonds,n.dekosta Washington: The National Academies .

7. T Nora, and Marry Pattman. " The solid networks development and the alliance for better citiesand communities.

8. UNICEF Innocenti Research Center. Building Child Friendly Cities: A structure for activity. Accessible.

9. WHO Collaborating Center on Society Safety Promotion. how-to-turn into anglobal safe-local area (got to on 21 December 2014).

10. Jat.inette - M.Mena my,John $t$ farerra,"Mediations for Self immolation Survivors: A Review of the Literature."Self immolationandLife ThreateningBehavior. 
The American Journal of Social Science and Education Innovations (ISSN - 2689-100x)

Published: April 28, 2021 | Pages: 184-189

Doi : https://doi.org/10.37547/tajssei/Volume03Issue04-27

2021: 5.857

11. Andrew s., Yokong, Heley y.pristor, and Mary K m.ford.Complicated distress and self-destructive ideating in grown-up overcomers of self immolation."Self immolationandLife Threatening

Behavior. 